\title{
Chlamydia trachomatis as a Cause of Neonatal Conjunctivitis in Dutch Infants
}

\author{
Ingrid G. I. J. G. Rours, MD, MMeda, Margaret R. Hammerschlag, MD', Alewijn Ott, MD, PhD', Tjeerd J. T. H. N. De Faber, MDd, \\ Henri A. Verbrugh, $\mathrm{MD}^{\mathrm{c}}$, Ronald de Groot, $\mathrm{MD}^{\mathrm{a}}$, Roel P. Verkooyen, $\mathrm{PhD}^{\mathrm{c}}$
}

aDepartment of Pediatrics, Sophia Children's Hospital, and 'Department of Medical Microbiology and Infectious Diseases, Erasmus MC University Medical Center, Rotterdam, Netherlands; bDepartment of Pediatrics, Division of Infectious Diseases, State University of New York Downstate Medical Center, Brooklyn, New York; dDepartment of Ophthalmology, Rotterdam Eye Hospital, Rotterdam, Netherlands

The authors have indicated they have no financial relationships relevant to this article to disclose.

\section{ABSTRACT}

BACKGROUND. Chlamydia trachomatis is the most common sexually transmitted pathogen in adults, which at delivery may be transmitted from mother to child and cause conjunctivitis and pneumonia. In the Netherlands, prenatal chlamydial screening and treatment of pregnant women is not routine practice. The contribution of $C$ trachomatis to neonatal ophthalmic disease has not been studied in the Netherlands and remains unclear.

METHODS. At the Sophia Children's Hospital and Rotterdam Eye Hospital, 2 cohorts of infants $<3$ months of age presenting with conjunctivitis were studied, 1 retrospectively (July 1996 to July 2001) and 1 prospectively (September 2001 to September 2002). Laboratory diagnosis was based on bacterial culture and polymerase chain reaction for $C$ trachomatis.

RESULTS. C trachomatis was detected in 27 (64\%) of 42 retrospectively studied infants and $14(61 \%)$ of 23 prospectively studied infants. Mucopurulent discharge was present in $35(95 \%)$ of 37 , swelling of the eyes in $27(73 \%)$ of 37, conjunctival erythema in $24(65 \%)$ of 37 , respiratory symptoms in $14(38 \%)$ of 37 , and feeding problems in $5(14 \%)$ of 37 infants respectively. Before microbiological diagnosis, general practitioners prescribed antichlamydial antibiotics locally to $5(12 \%)$ of 41 and systemically to $4(10 \%)$ of 41 infants who tested positive for chlamydia, and ophthalmologists prescribed to $21(51 \%)$ of 41 and 7 (17\%) of 41 , respectively.

CONCLUSIONS. C trachomatis was the major cause of bacterial conjunctivitis in this population. Clinically, differentiation from other pathogens was not possible. Many infants who tested positive for chlamydia did not receive appropriate antibiotic treatment.

$\mathrm{T}$ HE OCCURRENCE OF Chlamydia trachomatis infection in infants is directly related to the prevalence of maternal urogenital infections and vertical transmission rates. $^{1-4}$ The overall risk for infants born to women with untreated chlamydial infection is $\sim 50 \%$ to $75 \%$, with infection occurring at $\geq 1$ anatomic site. Conjunctivitis may occur in $20 \%$ to $50 \%$ of infected infants, nasopharyngitis in $\leq 70 \%$, and pneumonia in $5 \%$ to $20 \% .{ }^{5} \mathrm{C}$ trachomatis has become the most frequent identifiable cause of neonatal conjunctivitis in many countries. ${ }^{6-9}$ The majority of chlamydial conjunctivitis cases heal spontaneously during the first few months of life. However, untreated persistent infections can lead to acute discomfort and distress for both infant and mother, as well as to chronic eye disease. Simultaneous silent infection of the respiratory tract may cause acute or chronic respiratory disease.,3,10,11

Screening of pregnant women for $C$ trachomatis infection was recommended by the Centers for Disease Control and Prevention more than a decade ago.12,13 In 2004 the Dutch National Health Council advised against routine chlamydial screening of Dutch pregnant women, because local data with respect to chlamydial infection in pregnant 
women and the contribution of $C$ trachomatis to neonatal disease (pregnancy outcome, conjunctivitis, and respiratory tract infection) provided insufficient evidence to support screening. ${ }^{14,15}$ Recently we reported a prevalence of $C$ trachomatis infection in pregnant women of $6.4 \% .{ }^{16}$ Most of these infections had been missed in routine care, suggesting that $C$ trachomatis transmission to infants may often remain unnoticed.

The main objective of this study was to establish whether $C$ trachomatis was a cause of neonatal conjunctivitis in infants referred to hospital-based care in Rotterdam, the second largest city in the Netherlands. In addition, we evaluated the clinical presentation of and prescribed treatment for chlamydial conjunctivitis compared with other infections.

\section{MATERIALS AND METHODS}

\section{Study Population}

We conducted our study at the Rotterdam Eye Hospital (REH) and Sophia Children's Hospital (SCH) of the Erasmus University Medical Centre (Rotterdam, Netherlands). Rotterdam has a multiethnic population. Chlamydial screening is not standard practice in pregnant women in the study area; testing is done on clinical suspicion. Neonatal ocular prophylaxis against chlamydia or gonorrhoea is not routinely provided. Most newborns with conjunctivitis are treated at mother and child health clinics or by general practitioners (GPs). Infants with persistent conjunctivitis are referred to the $\mathrm{REH}$ or SCH depending on the parents' or referring GP's choice. We studied infants retrospectively between July 1996 and July 2001 and prospectively between September 2001 and September 2002. Infants $<3$ months of age presenting to the REH or SCH with bacterial (mostly persistent) conjunctivitis were eligible for the study. We defined bacterial conjunctivitis as having conjunctival erythema, swelling of the eyelids, and/or mucopurulent discharge. In the retrospective study, infants who were diagnosed by ophthalmologists with viral conjunctivitis (conjunctival erythema only) and who were neither microbiologically tested nor treated with antibiotics and improved spontaneously were excluded from the study. Similarly, infants with dacryostenosis (nasolacrimal duct obstruction) were excluded from the study. Infants diagnosed with chlamydial conjunctivitis in the REH were referred to the $\mathrm{SCH}$ for further investigation and systemic treatment. Infants diagnosed with $C$ trachomatis conjunctivitis in our prospective study were treated systemically with erythromycin suspension (ethylsuccinate), $50 \mathrm{mg} / \mathrm{kg}$ per day, in 3 to 4 oral doses for 10 to 14 days and were invited for a single follow-up visit. Parents were asked to return to the clinic if the infant had respiratory symptoms. Parents themselves were referred to the sexually transmitted disease clinic for investigation and treatment.

\section{Microbiological Diagnosis}

Eye swabs were taken for routine bacterial culture, including gonococcal culture, and chlamydial and gonococcal polymerase chain reaction (PCR) (Cobas Ampli- cor, Roche Molecular Diagnostics, Pleasanton, CA). Specimens were obtained by swabbing the conjunctiva of the everted lower eyelid using a sterile Dacron swab. Bacteriologic cultures were processed according to standard procedures for aerobic bacteria in a clinical microbiology laboratory. Chlamydial and gonococcal PCRs were performed according to the manufacturer's instructions.

\section{Treatment}

Antibiotics that were prescribed and considered to be effective against $C$ trachomatis were erythromycin, azithromycin, clarithromycin, tetracycline and doxycycline, and chloramphenicol.

\section{Data Collection}

We collected retrospective data through a systematic review of medical charts. We used a standardized questionnaire and laboratory investigations to collect prospective data. Recorded variables included the following: age at presentation, gender, complaints (conjunctival erythema, swelling of the eyelids, mucopurulent discharge, unilateral/bilateral involvement, and respiratory or feeding problems), physical examination, diagnostic tests, diagnosis, and therapy by GPs and ophthalmologists before microbiological diagnosis.

\section{Statistical Analysis}

We used SPSS 10.0.0 (SPSS Inc, Chicago, IL) for our analyses. We used uncorrected $\chi^{2}$ tests to compare categorical variables. We calculated risk ratios (with $95 \%$ confidence intervals) to examine factors associated with a diagnosis of chlamydia conjunctivitis.

\section{RESULTS}

\section{Demographics}

Retrospectively, 64 infants with conjunctivitis were identified; 36 (56\%) were boys. The median age was 2 weeks, with a range of 0 to 13 weeks. Prospectively, 23 infants were enrolled; 12 (52\%) were boys. The median age was 1 week, with a range of 0 to 7 weeks.

\section{Diagnostic Tests}

Five of 64 retrospectively studied infants were clinically diagnosed with bacterial conjunctivitis without performing a laboratory test. The remaining 59 infants had a bacterial culture done, including gonococcal culture; 42 infants also had a chlamydial PCR, and 17 infants had a gonococcal PCR. With inclusion of all of the tests, 50 $(85 \%)$ of 59 tested infants had a positive result. All 23 of the prospectively studied infants had bacterial and gonococcal cultures done, as well as a chlamydial and gonococcal PCR. Nineteen infants $(83 \%)$ had a pathogen detected. Table 1 shows the frequency of isolated species. Detection of $C$ trachomatis was significantly higher than of other pathogens $(P<.001)$, with similar rates in retrospectively and prospectively studied infants: 27 $(64 \%)$ of 42 and $14(61 \%)$ of 23 , respectively. Three infants in the retrospective cohort with Haemophilus influenzae had a second pathogen diagnosed. 
TABLE 1 Identified Pathogens Among Infants <3 Months of Age With Conjunctivitis

\begin{tabular}{|c|c|c|c|c|c|}
\hline $\begin{array}{l}\text { Pathogens Detected by } \\
\text { Test Method }\end{array}$ & $\begin{array}{c}\text { Retrospective } \\
\text { Cohort }\end{array}$ & $\begin{array}{c}\text { Prospective } \\
\text { Cohort }\end{array}$ & $\begin{array}{c}\text { Total } \\
\text { Infants }\end{array}$ & $P a$ & $\mathrm{RR}(95 \% \mathrm{Cl})^{\mathrm{a}}$ \\
\hline Bacterial culture, $n$ & 59 & 23 & 82 & & \\
\hline Staphylococcus aureus, n (\%) & $11(19)$ & $0(0)$ & $11(13)$ & & \\
\hline H influenzae, $n(\%)$ & $5(8)$ & $2(9)$ & $7(9)$ & & \\
\hline Streptococcus pneumoniae, $n(\%)$ & $4(7)$ & $0(0)$ & $4(5)$ & & \\
\hline N gonorrhoeae, $n(\%)$ & $2(3)$ & $0(0)$ & $2(2)$ & & \\
\hline Other pathogens, $n(\%)$ & $4(7)^{b}$ & $3(13)^{c}$ & $7(9)$ & & \\
\hline Cultures with pathogens, $n(\%)^{\mathrm{d}}$ & $24(41)$ & $5(22)$ & $29(35)$ & & \\
\hline PCR & 17 & 23 & 40 & & \\
\hline N gonorrhoeae, $n(\%)$ & $1(6)$ & $0(0)$ & $1(3)$ & & \\
\hline$P C R$ & 42 & 23 & 65 & & \\
\hline C trachomatis, $n(\%)$ & $27(64)$ & $14(61)$ & $41(63)$ & $<.001$ & $1.8(1.3-2.5)$ \\
\hline \multicolumn{6}{|c|}{$\begin{array}{l}\text { RR indicates risk ratio; Cl, confidence interval. } \\
\text { a The } P \text { value is for the difference in the yield between the nonchlamydia pathogen culture (29 of } 82 \text { ) and C trachomatis PCR ( } 41 \text { of } 65 \text { ). RR reflect } \\
\text { the higher likelihood of finding chlamydia than another pathogen in these infants. } \\
\text { b Other pathogens included Moraxella catarrhalis, Escherichia coli, Stenotrophomonas maltophilia, and hemolytic streptococcus. } \\
\text { cOther pathogens included } 2 \mathrm{M} \text { catarrhalis and } 1 \mathrm{~N} \text { meningitidis. }\end{array}$} \\
\hline
\end{tabular}

\section{Clinical Presentation}

We examined the age of 69 infants with microbiologically confirmed conjunctivitis. Of these infants, 41 had chlamydial conjunctivitis and 28 had another infection (Table 2 ). Infants with chlamydial conjunctivitis were 2.3 times (95\% confidence interval: 1.0-5.2 times) more likely than those with other infections to present between 1 and 6 weeks of age than within the first week of life. We were able to examine clinical information in 37 infants with chlamydial conjunctivitis (23 diagnosed retrospectively and 14 prospectively) and 22 with another pathogen (17 diagnosed retrospectively and 5 prospectively). Because there was no significant difference between the retrospective and prospective study, and the number of infants in each study was small, the overall results of a total of 37 evaluable infants who tested positive for chlamydia and 22 infants with another pathogen are shown in Table 2. Mu- copurulent discharge was the presenting symptom for 35 $(95 \%)$ of 37 infants who tested positive for chlamydia, swelling of the eyelids for 27 (73\%) of 37, and conjunctival erythema for $24(65 \%)$ of 37 compared with $22(100 \%)$ of $22,11(50 \%)$ of 22 , and $10(45 \%)$ of 22 , respectively, for infants with other pathogens; 27 (73\%) of 37 infants who tested positive for chlamydia had bilateral eye involvement compared with 17 (77\%) of 22 of infants with other pathogens. The presence of extraophthalmic symptoms did not differ between infants with chlamydia and those with another pathogen (Table 2). Feeding difficulties corresponded with respiratory complications in infants who tested positive for chlamydia. In the other group, these were separate cases. Additional symptoms in infants who tested positive for chlamydia included rhinitis (12 of 37), cough (4 of 37), excessive mucous ( 3 of 37 ), and wheezing or breathing

\begin{tabular}{|c|c|c|c|c|}
\hline Variable & $\begin{array}{c}\text { Ctrachomatis, } \\
n(\%)\end{array}$ & $\begin{array}{l}\text { Other Pathogens, } \\
n(\%)\end{array}$ & pa & $\mathrm{RR}(95 \% \mathrm{Cl})$ \\
\hline Age at presentation & 41 & 28 & .03 & \\
\hline$<1$ wk & $4(10)$ & $9(32)$ & & 1.0 (Reference) \\
\hline 1 to $6 w k$ & $34(83)$ & $15(54)$ & & $2.3(1.0-5.2)$ \\
\hline$>6 w k$ & $3(7)$ & $4(14)$ & & $1.4(0.4-4.5)$ \\
\hline No. of symptoms & 37 & 22 & .26 & \\
\hline 1 symptom & $8(22)$ & $9(41)$ & & 1.0 (Reference) \\
\hline MP discharge & $7(19)$ & $9(41)$ & & \\
\hline Redness & $1(3)$ & $0(0)$ & & \\
\hline 2 symptoms & $9(24)$ & $5(23)$ & & $1.4(0.7-2.6)$ \\
\hline MP discharge + swelling & $6(16)$ & $3(14)$ & & \\
\hline MP discharge + redness & $2(5)$ & $2(9)$ & & \\
\hline Redness + swelling & $1(3)$ & $0(0)$ & & \\
\hline $\begin{array}{l}3 \text { symptoms } \\
\text { MP discharge + swelling + redness }\end{array}$ & $20(54)$ & $8(36)$ & & $1.5(0.9-2.7)$ \\
\hline Extraophthalmic symptoms & 37 & 20 & & \\
\hline Respiratory & $14(38)$ & $6(30)$ & .55 & $1.3(0.6-2.8)$ \\
\hline Feeding & $5(14)$ & $2(10)$ & .70 & $1.4(0.3-6.4)$ \\
\hline
\end{tabular}

RR indicates risk ratio; $\mathrm{Cl}$, confidence interval; $\mathrm{MP}$, mucopurulent.

a The $P$ value was for the test of heterogeneity of age or number of symptom categories; the $P$ value of extraophthalmic symptoms was for the difference between pathogen categories. 


\begin{tabular}{llll} 
TABLE 3 & $\begin{array}{l}\text { Antibiotic Treatment Prescribed by GPs and } \\
\text { Ophthalmologists }\end{array}$ \\
\hline $\begin{array}{c}\text { Antibiotic Treatment According } \\
\text { to Diagnosis }\end{array}$ & $\begin{array}{c}\text { GPs, } n / N \\
(\%)\end{array}$ & $\begin{array}{c}\text { Ophthalmologists, } \\
n / N(\%)\end{array}$ & $P$ a \\
\hline Any confirmed bacterial conjunctivitis & & & \\
Any antibiotic & $30 / 69(43)$ & $51 / 69(74)$ & $<.001$ \\
Chlamydia conjunctivitis & & & \\
Any antibiotic & $20 / 41(49)$ & $31 / 41(76)$ & .01 \\
Antichlamydial antibiotic & $5 / 41(12)$ & $21 / 41(51)$ & $<.0001$ \\
Systemic antichlamydial antibiotic & $4 / 41(10)$ & $7 / 41(17)$ & .33 \\
\hline a The $P$ value is for difference in treatment choice between GPs and ophthalmologists.
\end{tabular}

difficulty ( 2 of 37 infants). In the group of infants with other pathogens, 4 of 20 had rhinitis; 1 had cough, and 1 had wheezing and crepitations.

\section{Therapy}

Both GPs and ophthalmologists prescribed topical antibiotics as eye ointment, gel, or drops, including tetracycline, aminoglycosides with or without steroids (gentamicin, soframycin, or tobramycin), fusidic acid, polymyxin/trimethoprim, ofloxacin, and erythromycin. In addition, GPs also prescribed chloramphenicol. Both GPs and ophthalmologists prescribed systemic treatment, including penicillin, clarithromycin, and erythromycin. In addition, GPs used cotrimoxazole and ophthalmologists used amoxicillin, augmentin, flucloxacillin, and cefotaxime. Antibiotic treatment by diagnosis as prescribed by GPs and ophthalmologists is shown in Table 3.

Comparison of treatment in the retrospective and prospective cohorts showed that ophthalmologists gave empiric antibiotics to $17(63 \%)$ of 27 infants who tested positive for chlamydia in the retrospective versus 14 $(100 \%)$ of 14 in the prospective study $(P<.01)$, antichlamydial antibiotics to $14(52 \%)$ of 27 and $7(50 \%)$ of 14 infants $(P=.9)$, and systemic treatment to $6(22 \%)$ of 27 and $1(7 \%)$ of 14 infants, respectively $(P=.22)$.

\section{Follow-up}

At follow-up, no more conjunctivitis was observed, and no symptoms or signs of respiratory tract infection were present.

\section{DISCUSSION}

This study demonstrated that $C$ trachomatis was the major cause of neonatal conjunctivitis in this population of infants referred to pediatric or eye hospitals in Rotterdam. On clinical presentation, no distinction could be made between chlamydial conjunctivitis and conjunctivitis caused by other pathogens. Empiric management to treat chlamydial conjunctivitis was frequently inappropriate.

The main limitation of this study was that we could only include infants with conjunctivitis who were referred to specialist hospitals. Other limitations were the incomplete diagnostic workup in the retrospective cohort and missing data on clinical symptoms in a few infants. Also, the number of children in analyses was relatively small, too small to draw firm conclusions.
Although we cannot show what proportion of all neonatal conjunctivitis is caused by $C$ trachomatis, we have shown that it is an important cause of (persistent) conjunctivitis. Determining the incidence of chlamydial neonatal conjunctivitis and transmission rates would require a prospective study testing all pregnant women and their newborns.

The identification rate of pathogenic bacteria in our study was $84 \%$, which is higher than in most reports. ${ }^{17-20}$ In addition, $C$ trachomatis was the organism isolated most often. This is likely to be related to our study design and to the Dutch referral system, in which mainly infants with persistent conjunctivitis are referred to ophthalmologists. However, we may even have underestimated the contribution of chlamydia to persistent neonatal conjunctivitis, because we excluded the infants with a clinical diagnosis of viral conjunctivitis. Underdiagnosis of $C$ trachomatis persistent conjunctivitis may also be suggested by the relatively low observed number of 5 cases per year in the retrospective study versus 14 in the prospective year. However, the latter increase may also reflect an actual increase of chlamydial infection in Dutch adults. ${ }^{21}$

To put our results into context, we related the findings from our prospective study to obstetric data from the SCH in that year. There were 1648 deliveries in total, of which 731 were in women $<30$ years of age. With an antenatal prevalence of $6.4 \%, 16 \sim 47$ women would be expected to have $C$ trachomatis infection. With an estimated transmission rate of $50 \%$ to $75 \%$ and $20 \%$ to $50 \%$ of these developing conjunctivitis, ${ }^{5}$ we would expect 9 to 24 infants with chlamydial conjunctivitis, which corresponds with our findings. This may suggest that all of the infants with conjunctivitis were detected. However, not all of the studied infants were born to women delivering at the $\mathrm{SCH}$, but also in other hospitals in Rotterdam or at home.

The clinical presentation of chlamydial conjunctivitis has been extensively described by others, and our study shows similar results to previous reports.,3,4,922-24 Most infants who tested positive for chlamydia presented between the ages of 1 and 6 weeks (because of the slow reproductivity of the organism) and with all 3 of the symptoms (erythema, swelling, and discharge).

Ophthalmologists more often prescribed antibiotics that were effective against chlamydial infection than GPs. Still, only half of the infants with chlamydial conjunctivitis received effective antibiotics before microbiological diagnosis, and $<20 \%$ received systemic treatment. In the prospective study, even fewer infants received systemic treatment. This probably reflects the agreement made with ophthalmologists to refer infants to a pediatrician after diagnosis. We cannot draw any conclusions about chlamydial conjunctivitis in primary health care from our study. Acute conjunctivitis may be correctly treated by GPs with topical antibiotics according to Health Care Insurance Board guidelines. However, chlamydia is probably not being considered in the differential diagnosis by some GPs, because only $12 \%$ of infants with chlamydia had received antibiotics that were active against $C$ trachomatis. 
Our results may suggest the need to institute eye prophylaxis for ophthalmia neonatorum, which is not practiced in the Netherlands. However, we do not want to advocate the application of neonatal eye prophylaxis, because prophylaxis does not prevent all chlamydial neonatal conjunctivitis, and the absence of conjunctivitis as an indicator of chlamydial infection may delay the proper diagnosis of (silent) chlamydial infection at other sites. Another motivation against the decision to start routine eye prophylaxis is that this may lead to overtreatment of newborns in a country with easy access to medical care. We would rather like to use our findings to indicate the need for proper (systemic) antibiotic treatment of newborns who test positive for chlamydia and screening of pregnant women.

Chlamydial screening for pregnant women is not standard practice in the study area or in the rest of the Netherlands. Testing is only done when clinically warranted. In the prospective year of the study, only $1 \%$ of 1648 deliveries were tested for $C$ trachomatis, of which 4 tested positive. These figures may also reflect the underestimation of chlamydial infection in pregnant women. The value of screening may warrant additional discussion. ${ }^{16}$ To prevent 1 case of chlamydial conjunctivitis, 31 to 78 pregnant women need to be screened. Assuming a specificity of $\geq 99.8 \%,{ }^{16}$ this could result in 0.06 to 0.16 false-positive mothers and 2 to 5 truly positive mothers (and their partners); furthermore, this could result in the prevention of 1.4 to 2.5 nasopharyngitis cases and 0.2 to 1.0 pneumonia cases. Previously we described the costs of chlamydia screening of pregnant women by different DNA-isolation methods in individual and pooled urine samples. ${ }^{16}$ The cost per chlamydia case detected when using the Cobas Amplicor on individual urine samples was $€ 275$ and when using a combined method of isolation with the MagNA Pure bacterial DNA-isolation kit (Roche Molecular Diagnostics, Pleasanton, CA), and subsequent amplification and detection by Cobas Amplicor on pooled urine samples, as we did, was €108.

In the absence of a chlamydial screening program, we urge clinicians to have a higher index of suspicion for neonatal and maternal chlamydial infection. Furthermore, we recommend that, in areas where prenatal chlamydial screening and treatment of pregnant women is not routine practice, infants with signs of conjunctivitis that persist for $>72$ hours while applying frequent normal saline eye irrigation should have a full microbiological evaluation including Neisseria gonorrhoeae and C trachomatis. While awaiting laboratory results, empiric treatment in this population should include erythromycin eye drops or ointment to both eyes. We recommend that confirmed chlamydial conjunctivitis should be treated systemically with erythromycin suspension or alternatively azithromycin. ${ }^{13,25}$

Current national treatment guidelines for neonatal chlamydial conjunctivitis are from the Health Care Insurance Board and recommend tetracycline eye drops (with or without oral erythromycin or tetracycline). ${ }^{26}$ The current Centers for Disease Control and Prevention recommendation, however, is to treat all neonatal chlamydial conjunctivitis systemically with a 14-day course of erythromycin suspension. ${ }^{13}$ Systemic treatment has been demonstrated to be more effective than topical treatment, and infants with conjunctivitis are often infected at other sites as well. ${ }^{27}$

\section{CONCLUSIONS}

The high rate of isolation of $C$ trachomatis in infants with persistent conjunctivitis confirms the importance of this infection in the Netherlands. GPs and ophthalmologists need to consider chlamydia in the differential diagnosis of neonatal conjunctivitis and use appropriate antibiotics. Targeted screening for $C$ trachomatis prenatally to prevent infection and related complications in both mother and infants should again be considered in those countries where it is not routine practice.

\section{ACKNOWLEDGMENTS}

We thank Martina Bronner (REH) and Connie Groenendijk and Mirjam van Veen (SCH) for assisting with the logistics between centers.

\section{REFERENCES}

1. Bell TA, Stamm WE, Kuo CC, Wang SP, Holmes KK, Grayston JT. Risk of perinatal transmission of Chlamydia trachomatis by mode of delivery. J Infect. 1994;29(2):165-169

2. Schachter J, Sweet RL, Grossman M, Landers D, Robbie M, Bishop E. Experience with the routine use of erythromycin for chlamydial infections in pregnancy. $N$ Engl J Med. 1986;314(5): 276-279

3. Preece PM, Anderson JM, Thompson RG. Chlamydia trachomatis infection in infants: a prospective study. Arch Dis Child. 1989; 64(4):525-529

4. Alexander ER, Harrison HR. Role of Chlamydia trachomatis in perinatal infection. Rev Infect Dis. 1983;5(4):713-719

5. Hammerschlag MR. Chlamydial infections. J Pediatr. 1989; $114(5): 727-734$

6. Laga M, Plummer FA, Nzanze H, et al. Epidemiology of ophthalmia neonatorum in Kenya. Lancet. 1986;2(8516): 1 145-1149

7. Sergiwa A, Pratt BC, Eren E, Sunona TC, Hart CA. Ophthalmia neonatorum in Bangkok: the significance of Chlamydia trachomatis. Ann Trop Paediatr. 1993;13(3):233-236

8. Sandstrom I. Etiology and diagnosis of neonatal conjunctivitis. Acta Paediatr Scand. 1987;76(2):221-227

9. Dannevig L, Straume B, Melby K. Ophthalmia neonatorum in northern Norway. II. Microbiology with emphasis on Chlamydia trachomatis. Acta Ophthalmol (Copenh). 1992;70(1):19-25

10. Mordhorst CH, Dawson C. Sequelae of neonatal inclusion conjunctivitis and associated disease in parents. Am J Ophthalmol. $1971 ; 71(4): 861-867$

11. Harrison HR, English MG, Lee CK, Alexander ER. Chlamydia trachomatis infant pneumonitis: comparison with matched controls and other infant pneumonitis. N Engl J Med. 1978; 298(13):702-708

12. Centers for Disease Control and Prevention. Sexually transmitted diseases treatment guidelines [published correction appears in MMWR Morb Mortal Wkly Rep. 1989;38(38):664]. MMWR Morb Mortal Wkly Rep. 1989;38(suppl 8):1-43

13. Centers for Disease Control and Prevention. Sexually transmitted diseases treatment guidelines [published correction appears in MMWR Recomm Rep. 2006;55(36):997]. MMWR Recomm Rep. 2006;55(RR-11):1-94

14. Bosch WJHMvd. Chlamydia Trachomatis. The Hague, Netherlands: Gezondheidsraad; 2003 
15. van de Laar MJW. SOA en AIDS in Nederland. Bilthoven, Netherlands: Rijksinstituut voor Volksgezondheid en Milieu; 2000

16. Rours GI, Verkooyen RP, Willemse HF, et al. Use of pooled urine samples and automated DNA isolation to achieve improved sensitivity and cost-effectiveness of large-scale testing for Chlamydia trachomatis in pregnant women. J Clin Microbiol. 2005;43(9):4684-4690

17. Sandstrom KI, Bell TA, Chandler JW, et al. Microbial causes of neonatal conjunctivitis. J Pediatr. 1984;105(5):706-711

18. Fransen L, Van den Berghe P, Mertens A, Van Brussel K, Clara $\mathrm{R}$, Piot P. Incidence and bacterial aetiology of neonatal conjunctivitis. Eur J Pediatr. 1987;146(2):152-155

19. Di Bartolomeo S, Mirta DH, Janer M, et al. Incidence of Chlamydia trachomatis and other potential pathogens in neonatal conjunctivitis. Int J Infect Dis. 2001;5(3):139-143

20. Persson K, Ronnerstam R, Svanberg L, Pohla MA. Neonatal chlamydial eye infection: an epidemiological and clinical study. Br J Ophthalmol. 1983;67(10):700-704
21. Kolader ME, Thiesbrummel HFJ, Peetam E. Year Report STD Clinic 2004. Amsterdam, Netherlands: Municipal Health Services Amsterdam; 2004

22. Hammerschlag MR. Neonatal conjunctivitis. Pediatr Ann. 1993; 22(6):346-351

23. Stenberg K, Mardh PA. Chlamydial conjunctivitis in neonates and adults: history, clinical findings and follow-up. Acta Ophthalmol (Copenh). 1990;68(6):651-657

24. Hammerschlag MR, Chandler JW, Alexander ER, English M, Koutsky L. Longitudinal studies on chlamydial infections in the first year of life. Pediatr Infect Dis. 1982;1(6):395-401

25. Hammerschlag MR, Gelling M, Roblin PM, Kutlin A, Jule JE. Treatment of neonatal chlamydial conjunctivitis with azithromycin. Pediatr Infect Dis J. 1998;17(11):1049-1050

26. Loenen AC. Farmacotherapeutical Kompass. Amstelveen, Netherlands: Health Care Insurance Board; 2005

27. Patamasucon P, Rettig PJ, Faust KL, Kusmiesz HT, Nelson JD. Oral v topical erythromycin therapies for chlamydial conjunctivitis. Am J Dis Child. 1982;136(9):817-821 